\title{
Creatine Supplementation Effect on Recovery Following Exercise-Induced Muscle Damage: A Systematic Review and Meta-analysis of Randomized Controlled Trials
}

\author{
Liu Gang ${ }^{1}$ \\ ${ }^{1}$ Hunan University of Technology
}

September 16, 2020

\begin{abstract}
Exercise-induced muscle damage (EIMD) causes to increased soreness, impaired function of muscles, and reductions in muscle force. Accumulating evidence suggests beneficial effects of creatine on EIMD. Nevertheless, outcomes differ substantially across various articles. The main aim of this meta analysis was to evaluate the effect of creatine on recovery following EIMD. Medline, Embase, Cochrane Library, Scopus and Google scholar were systematically searched up to July 2020. The Cochrane Collaboration tool for examining risk of bias was applied for assessing the quality of studies. Weighted mean difference (WMD), 95 percent confidence interval (CI) and random effects model, were applied for estimating the overall effect. Between studies heterogeneity was examined using the chi-squared and I2 statistic. Nine studies met the inclusion criteria. Pooled data showed that creatine significantly reduced CK concentration overall (WMD $=-30.94 ; 95 \%$ CI: $-53.19,-8.69 ; \mathrm{P}=0.006)$ and at three follow-up times 48, 72 and 96 hours) in comparison with placebo. In contrast, effects were not significant in LDH concentration overall (WMD $=-5.99 ; 95 \%$ CI: $-14.49,2.50 ; \mathrm{P}=0.167$ ), but creatine supplementation leaded to a significant reduction in LDH concentrations in trials with 48 hours measurement of LDH. The current data indicates that creatine consumption is better than rest after diverse forms of damaging and exhaustive exercise or passive recovery. The benefits relate to a decrease in muscle damage indices and Improved muscle function because of muscle power loss after exercise.
\end{abstract}

Text-only word count: 3286

Number of Tables: 3

Number of Figures: 4

\section{Abstract}

Exercise-induced muscle damage (EIMD) causes to increased soreness, impaired function of muscles, and reductions in muscle force. Accumulating evidence suggests beneficial effects of creatine on EIMD. Nevertheless, outcomes differ substantially across various articles. The main aim of this meta analysis was to evaluate the effect of creatine on recovery following EIMD. Medline, Embase, Cochrane Library, Scopus and Google scholar were systematically searched up to July 2020. The Cochrane Collaboration tool for examining risk of bias was applied for assessing the quality of studies. Weighted mean difference (WMD), 95 percent confidence interval (CI) and random effects model, were applied for estimating the overall effect. Between studies heterogeneity was examined using the chi-squared and $\mathrm{I}^{2}$ statistic. Nine studies met the inclusion criteria. Pooled data showed that creatine significantly reduced CK concentration overall (WMD $=-30.94 ; 95 \%$ CI: $-53.19,-8.69 ; \mathrm{P}=0.006$ ) and at three follow-up times 48,72 and 96 hours) in comparison with placebo. In contrast, effects were not significant in $\mathrm{LDH}$ concentration overall $(\mathrm{WMD}=-5.99 ; 95 \%$ CI: $-14.49,2.50 ; \mathrm{P}=$ 0.167), but creatine supplementation leaded to a significant reduction in LDH concentrations in trials with 48 hours measurement of LDH. The current data indicates that creatine consumption is better than rest 
after diverse forms of damaging and exhaustive exercise or passive recovery. The benefits relate to a decrease in muscle damage indices and Improved muscle function because of muscle power loss after exercise.

Keywords: Creatine; Creatine Kinase; Lactate Dehydrogenase; Muscle Damage; Meta-analysis; Systematic Review

\section{Introduction}

It is well known that various types of exercise induce muscle damage. Exercise-induced muscle damage (EIMD) is recognized as the outcome of intense to moderate physical activity $(1,2)$. This damage manifests itself as a temporary decrease muscle function, increases in serum creatine kinase (CK) and lactate dehydrogenase (LDH) concentrations, increases in myoglobin and delayed onset muscle soreness (DOMS) (3). The basic feature of skeletal muscle damage without cell necrosis is the muscle fiber disruptions, chiefly the basal lamina sheath. Regarding mechanical stimuli, especially intense physical activity, it is understood that it can increase micro damage in muscle fibers imposed by eccentric contractions, according to the volume and length the damage degree and DOMS may be persist chronically over time $(4,5)$. The symptoms of DOMS which include muscle pain, strength loss, tenderness, swelling and stiffness have been suggested to happen within 24 hours of muscle damage and last beyond five days (6). Degradation of contractile proteins appears to relate to reduces in muscular force about one week to one month after eccentric exercise (7). Due to EIMD and DOMS that follows intense, unaccustomed or eccentrically biased exercise, a focus on recovery strategies that attenuate these unwanted effects should be pursued. Recently, some studies indicated that creatine consumptin might reduce post-exercise muscle damage via mechanisms regulating mitochondrial permeability and stabilizing the sarcolemma (8-10).

Newly, many athletes have consumed ergogenic aids to enhancing recovery, maintain body conditioning and physiological adaptations during long-term exercise programmes. Thus, the efficacy of ergogenic aids has always attracted great attention, and many researchers have sought to combine exercise training programmes and ergogenic aid to reinforce the benefits of exercise $(11,12)$. Creatine is a popular and favorite ergogenic aid at all levels among athletes that is synthesized endogenously from the amino acids glycine, methionine and arginine or intaked from the diet $(8,13)$. Articles studing the creatine supplementation effects have demonstrated improved performance in various exercise under a variety of different testing situation (14, $15)$, although this has not been shown in all studies $(16,17)$. Theoretically, enhancing the availability of phosphocreatine would augment cellular bioenergetics of the phosphagen system $(18,19)$ in addition to the high-energy phosphates shuttling between the cytosol and mitochondria via the creatine phosphate shuttle (20). Also, there is evidence that supplementation with creatine may provide benefits for patients with diversity of neuromuscular diseases $(21,22)$ and metabolic disorders $(23,24)$. These results have showed that creatine supplementation may provides a ergogenic aid for individuals and also may present some therapeutic advantage for special populations.

In this regard, all related articles have been gathered in this study and as far as the studies quality allows and the creatine effects on EIMD have been evaluated. This systematic review and meta-analysis summarizes a detailed analysis about the hypothesis that creatine consumption can ameliorate EIMD and DOMS.

\section{Methods}

\section{Search Strategy}

The systematic review and meta-analysis was conducted based on Preferred Reporting Items for Systematic Reviews and Meta-Analyses (PRISMA) (25). Electronic and manual search was performed up to July 2020 in Embase, Medline, Cochrane Library, Scopus and a supplementary search in Google Scholar. The following medical subject headings (MeSH) and non-MeSH terms keywords were applied, including: "creatine", "creatine monohydrate", "creatine supplementation", "creatine loading", "exercise", "physical exercise", "eccentric exercise", "aerobic exercise", "athlete", "muscle damage" "creatine kinase", "lactate dehydrogenase", "controlled trial", "randomized", "randomised", "random", "randomly", "randomized clinical trial", "RCT", "blinded", "double blind", "double blinded", "trial", "controlled clinical trial", "crossover proce- 
dure", "cross-over trial", "double blind procedure "and "equivalence trial". References lists of all studies and previous review articles were screened for more eligible studies identification.

\section{Eligibility Criteria}

Articles were elected using criteria of Population-Intervention-Comparator-Outcomes-Study design (PICOS) (25) including: 1) healthy subjects received oral creatine supplementation, as a nutritional strategy; 2) original randomized-controlled trial researches; 3) reporting at least one measurement of muscle damage indices (creatine kinase $(\mathrm{CK})$ and lactate dehydrogenase $(\mathrm{LDH})$ ); 4) provided muscle damage indices data as mean and standard deviation (SD) in both intervention and placebo groups. Also, criteria for exclusion were: 1) using a mixture of creatine in intervention group only (vitamins, Coenzyme Q10 and etc.); 2) semi experimental, trials without control groups and nonrandomized trials; 3) animal studies; 4) reviews and case reports

\section{Selection strategy:}

After a primary search, all extracted studies found from searches were uploaded into endnote software (EndNote X6, Thomson Reuters, New York) for screening. Titles and abstracts of papers were screened using the search strategy. Articles were evaluated independently by two authors and selected according to the inclusion criteria. Studies including eligibility criteria in the screening of title and abstract were elected to be checked by full-text. On the basis of the qualified data, we used a standardized form to elect the all categorized studies eligible for inclusion in this review. Contradictions between the authors were resolved by third researcher or consensus.

\section{Data extraction}

We extracted following data by a standardized electronic form (Microsoft Office, Excel sheets): first author's name, publication year and country, design of study, gender and age of subjects, sample size, intervention duration and creatine dose. Also, we extracted mean and SD of plasma CK and LDH at baseline and after the intervention. Any recorded standard errors of mean (SEM), were converted to SDs through this formula: $(\mathrm{SD}=\mathrm{SEM} \times[?] \mathrm{n}(\mathrm{n}=$ number of individuals in intervention or placebo groups). At last, in papers that reported information in figures, extraction was carried out using GetData Graph Digitizer 2.24 (26).

Study quality

As regards it has been adopted that study inclusion with a high risk of bias can distort the meta-analysis outcomes $(25,27)$, the Cochrane Collaboration tool was used for evaluating the bias risk. The included studies quality were evaluated by following items: randomization sequence generation; allocation concealment; participants, personnel, assessor and investigator blinding; financial interest by companies and attrition rates. These items were given a rating of high, low, or unclear risk of bias. A randomized controlled trial (RCT) was categorized as having overall high, low or medium risk based on the basic items of allocation concealment, participants and assessor blinding, and reporting of attrition rates (High $=$ High risk of bias for one or more than one key areas, Low = Low risk of bias for all key areas, and Medium = Unclear or low risk of bias for all key areas).

Analyses and measures of treatment effect

This meta-analysis was planed to extract information based on differences of baseline and follow up times for every trial treatment (mean change data); nonetheless, most articles reported follow-up data in figures and we extracted follow-up times mean differences and SD. We applied standardized mean differences for variables pooled on the different scales. Heterogeneity was evaluated between studies by the Cochrane's $\mathrm{Q}$ and quantified by the $\mathrm{I}^{2}$ statistic, that demonstrated percentage of the total variation through articles that is ascribable to heterogeneity rather than to chance. P-value of $<0.05$ and level of $\mathrm{I}^{2}>40$ percent was considered as significant heterogeneity.

For estimating the overall effect, we computed the weighted mean differences (WMDs) with 95 percent confidence intervals (CIs) using random effects model. To measure if the outcomes could have been affected 
by a single study on the overall distinctly, a sensitivity analysis was performed (28). Subgroup analysis was also conducted, based on follow-ups $(<24,24,48,72$ and 96 hours after exercise), dose of creatine (20 g/day or lower and more than $20 \mathrm{~g} /$ day), duration of studies (1 week and lower and more than 1 week) and train status (trained and untrained). For evaluating the publication bias we applied visual inspection of funnel plots, Egger's regression asymmetry and means of Begg's rank correlation test. Funnel plots depicted the effect sizes (differences in means) against their corresponding standard errors. Also, statistical analyses were conducted applying STATA 11.2 software (StataCorp, College Station, Texas, USA).

\section{Results}

Search outcomes and included articles overview

Our search led to 850 related studies. After duplicates removing $(\mathrm{n}=27)$, a wide screening range of the titles and abstracts and careful assessment was conducted on 823 related studies. Of these, 29 studies remained after considering the inclusion and exclusion criteria for the eligibility. Finally, 9 studies, including, 30 effect sizes for CK and 24 effect sizes for LDH, which studied a total of 630 and 420 participants respectively and aged $19.9-37.5$ years, were identified in the current review. This numbers is inclusive of individuals who were dropouts in some experiments. Participants tended to be young, but one study evaluated creatine effects on CK and LDH concentration for middle-aged men in addition to youth (29). Moreover, all participants were men, except in one study which women participated $(\mathrm{n}=15)(30)$, and in one study both men and women participated $(\mathrm{n}=49)(31)$.

Two studies were not randomized $(32,33)$ and data from two studies was not directly accessible from the published papers, and we sent email and request the raw data but we did not receive information from the studies authors $(34,35)$. Figure 1 shows the selection procedure and causes for excluding the studies and the data inTable 1 illustrates the basic characteristics of the articles in our systematic review and meta-analysis.

In brief, the studies were published between 1998 and 2018. The total number of individuals who completed the studies in inclusion criteria was 319 and 311 individuals in the intervention and placebo groups respectively for CK and 211 and 209 individuals in the intervention and placebo groups respectively for LDH. The dose of creatine supplementation was 2 to $40 \mathrm{~g}$ /day among these studies and the duration of these trials ranged between 5 to 56 days. All studies used a randomized parallel design, and all of them had the design of double-blind except two studies $(36,37)$. The effect of creatine on CK and LDH concentration together was examined in 5 studies (29, 38-41) and 4 studies only reported CK $(30,31,37,42)$.

Some of the articles undertook multiple follow-up (e.g., muscle damage indices for intervention and placebo immediately, 0.5, 1, 2, 4, 24, 48, 72, and 96 hour and 7, 10, 14, 21 days after exercise). We focused on results reported immediately post exercise ( $<24$ hours) and subsequent hours (24, 48, 72 and 96 hours). Eight trials in 4 studies reported $<24$ hours follow-up times $(30,31,39,42)$; Five trials in 4 studies had 24 hours follow-up times (38-40, 42); Seven trials in 6 studies reported 48 hours follow-up times (29, 37-40, 42); Four trials in 4 studies reported follow-ups at 72 hours after exercise $(29,38-40)$ and four trials in 4 studies reported follow-ups at 96 hours after exercise (37-40).

Results from quality assessments

Table 2 reported the quality details of bias assessment. Briefly, participants random allocation was not mention in all included articles except in one study that explained the procedure of random sequence generation (31). All of 9 articles did not report allocation concealment. Most of articles represented low risk of bias for selective reporting; nonetheless, 2 articles had high risk of bias $(30,39)$ and 2 articles had unclear risk of bias $(29,37)$. All articles had a low risk of bias based on incomplete outcome data. Most of articles had a unclear or high risk of bias for blinding of personnel and participants except just one article that had low risk of bias as to participants and personnel blinding (31). Also, all of articles had a high or unclear risk for blinding outcome assessors. At last, about other potential threats to validity, all of articles had low risk of bias. 
Meta-analysis findings

Effects of creatine supplementation on serum CK concentration

According to our analysis on 25 trials, in overall, creatine consumption had significant effect on CK concentration: $(\mathrm{WMD}=-30.94 ; 95 \% \mathrm{CI}:-53.19,-8.69 ; \mathrm{P}=0.006)$. There was significant heterogeneity among the articles $\left(\mathrm{P}=0.000, \mathrm{I}^{2}=99.8 \%\right)$ (Figure 2). Subgroup analysis was coducted to investigate if the effect of creatine supplementation on serum CK is different according to follow-ups after exercise, dose of creatine, duration of studies and train status (Figure $\mathbf{2}$ and Table $\mathbf{3}$ ). Some of subgroup analysis showed that creatine consumption resulted in a significant reduction in CK concentrations in trials with 48,72 and 96 hours measurement of CK after exercise, >20 g/day creatine consumption, [?]1 trial duration and studies on untrained participant.

Effects of creatine supplementation on LDH concentration

As outlined in Figure 3 and Table $\mathbf{3}$, our preliminary analysis indicated that creatine supplementation have no significant effect in serum LDH concentration compared to placebo (WMD $=-5.99 ; 95 \%$ CI: 14.49, 2.50; $\mathrm{P}=0.167)$. Also, significant heterogeneity was observed among studies $\left(\mathrm{P}=0.014, \mathrm{I}^{2}=47.2\right.$ $\%)$. Some of subgroup analysis showed that creatine consumption resulted in a significant reduction in $\mathrm{LDH}$ concentrations in trials with 48 hours measurement of LDH after exercise and >20 g/day creatine consumption.

Sensitivity analysis and publication bias

Sensitivity analysis indicated that any removal of the studies from the meta-analysis, create no change in the results of the meta-analysis on CK and LDH concentration. Funnel plots for CK and LDH were visually symmetrical (Figure 4), and the Begg's test results did not determine any publication bias evidence in articles that examine the effect of creatine supplementation on CK (Begg's test, P $=0.176$ ) and on LDH (Begg's test, $\mathrm{P}=0.185$ ).

\section{Discussion}

The results of the current meta-analysis, performed on 25 treatment arms, showed advantageous effects of creatine consumption in reduction of CK and LDH concentration after different follow-ups after exercise.

Muscle Damage is a phenomenon that may happen because of several reasons, like cell necrosis or rupture, including about 10 to 55 percent of total muscular injuries (43). The muscle damage can be determined as the plasma membrane disruption, accompanied by the muscle proteins loss (i.e. CK, LDH and myoglobin), increased inflammatory cells crowd in the muscle fibers (i.e. neutrophils and macrophages), influx of serum proteins, DOMS, functional impairment (week performance and loss of strength), and some structural disorders like sarcomere $\mathrm{Z}$ lines disarrangement $(7,44)$.

The present meta analysis evaluated indirect muscle damage indices, including CK and LDH among trained and untrained both sexes individuals, looking at reported articles. Proteins like CK and LDH have been extensively applied as markers of muscle micro-damages (45) and usually has been evaluated to be an indirect biomarkers of post exercise muscle damage due to their easiness of identification and the relatively low cost of tests to quantify it $(46,47)$. In spite of CK and LDH rising post exercise being much variable and affected by exercise variable and individual factors, the assessment of CK and LDH pre- and post-exercise may provide a diagnostic tool for the detection of post-exercise muscle damage, with much less invasiveness than needed in a muscle biopsy (48).

Wide assays has assessed the creatine consumption effects on performance in exercise (12). However, a few surveys have assessed the effect of creatine consumption on muscle recovery post exercise damage (49, 50). Willoughby and Rosene (51) have indicated that by ongoing creatine consumption post exercise, creatine may operate as a gene transcription co-regulator of amino acid pools, therefore increasing synthesis of myofibrillar protein during recovery after exersice (52). 
Almost 95 percent of creatine is accumulated in skeletal muscles while the remained 5 percent is stored the brain, testes and heart $(53,54)$. Of this, about 60 percent of creatine is accumulated as phosphocreatine with the remaining creatine containing the free creatine pool (55). Creatine consumption (e.g. $20 \mathrm{~g} /$ day for 5 days) has been reported to enhance phosphocreatine and muscle free creatine usually by 15 to 40 percent (56). It is indicated that creatine, can bind to the plasma membrane due to amphipathic nature, therewith increasing membrane stability and decrease CK and LDH loss (57).

In the current meta-analysis the positive effects of creatine could cause decrease in CK concentration overall, regardless of subgroup analysis, while being non-significant in LDH. Subgroup analysis showed the creatine supplementation effect on lowering serum CK is significant in 48, 72 and 96 hours follow-ups after exercise. But due to high heterogeneity of the data to our knowledge these results should not be considered reliable. Also, creatine consumption reduce LDH significantly in 48 hours follow-ups after exercise and there was no considerable heterogeneity between trials. CK and LDH responses might depend on when the initial muscle damage happened, the training status of the individuals and the orientation with the physical activity applied (58), and thus the limit of myocellular specific proteins release. In this regard, trials with untrained participants had a significant decrease in CK concentrations with creatine supplementation.

According to expectations, dose of $>20 \mathrm{~g}$ /day creatine had significant effect on lowering CK. But contrary to expectations trials with creatine supplementation duration of less than one week had significant lower CK concentration. It can be speculated that participant in all 8 trials ( 2 studies) that used $>20 \mathrm{~g} /$ day creatine, conducted with duration of less than one week $(31,39)$. Above-mentioned subgroup analysis indicated that dose of creatine supplementation is more important than duration of supplementation for decreased CK concentration.

Weight gain is the only side effect that has been suggested which may be a desirable effect for many patient populations and athletes (59-61). Contrary to this safety, worries have been increased in scientific community and media related to the creatine consumption (62-64). In this case worries have been increased that consumption of creatine may elevate chronically creatine synthesis suppression, promote muscle, liver and renal damage, alter electrolyte situation and lead to long-term side effects $(63,65,66)$. Moreover, creatine has been reported to cause dehydration, diarrhea, promote cramping and increase gastrointestinal upset (67-69).

The present meta-analysis had several limitations. No included studies in this meta-analysis blinded the providers/ assessors and only one study blinded the participants. Due to the nature of physical activity interventions, blinding in such studies may be challenging. Moreover, evidence was downgraded due to the lack of homogeneity among included articles and subgroup hypothesis were not sufficient for founding the source of heterogeneity. However, follow-ups after exercise explained potential between-study heterogeneity, especially for LDH concentration. Lack of information about data on intensity and frequency of exercise, genetic background, lifestyle factors and lack of complete baseline CK and LDH data for subgroup analysis make overall interpretation of the results difficult. Further, a significant gender affect was suggested in activity of serum CK concentration in other investigations (70). Many factors can influence the high heterogeneity stated.

The main strengths of the current meta-analysis are evaluation of $<24,24,48,72$ and 96 hours follow-ups measurement of muscle damage indices after exercise and subgroup analysis based on follow-ups, the dose of supplementation, trial duration and train status of individuals on the overall effect sizes. The second one, we endeavored to minimize any biases in this meta-analysis procedure by conducting a comprehensive search of the databases as well as performing and describing the results using the PRISMA guidelines.

\section{Conclusion}

In summary, the findings within the current meta analysis show that creatine supplementation would be effective in reducing the immediate muscle damage that happens post exercise muscle injury. But, due to high heterogeneity and the medium risk of bias for articles, we suggest that these results be taken into account and the facts be interpreted with caution by the readers. Further research with diverse dosages of 
creatine is required to know the optimal dose and the best repetition per day for more efficient recovery.

\section{Acknowledgments}

We are grateful to all the participants in the study and to Hunan University of Science and Technology for funding the study.

Funding: This work was supported by Hunan University of Science and Technology

Competing interests: authors have no competing interests to declare

\section{References}

1. Camera DM, Smiles WJ, Hawley JA. Exercise-induced skeletal muscle signaling pathways and human athletic performance. Free Radical Biology and Medicine. 2016;98:131-43.

2. Chazaud B. Inflammation during skeletal muscle regeneration and tissue remodeling: application to exercise-induced muscle damage management. Immunology and cell biology. 2016;94(2):140-5.

3. Harty PS, Cottet ML, Malloy JK, Kerksick CM. Nutritional and supplementation strategies to prevent and attenuate exercise-induced muscle damage: a brief review. Sports medicine-open. 2019;5(1):1.

4. Avery NG, Kaiser JL, Sharman MJ, SCHEETT TE, Barnes DM, Gomez AL, et al. Effects of vitamin E supplementation on recovery from repeated bouts of resistance exercise. The Journal of Strength \& Conditioning Research. 2003;17(4):801-9.

5. Kashef M. Effect of vitamin E supplementation on delayed onset muscle soreness in young men. Journal of Physical Activity and Hormones. 2018;2(3):15-28.

6. Heiss R, Lutter C, Freiwald J, Hoppe MW, Grim C, Poettgen K, et al. Advances in Delayed-Onset Muscle Soreness (DOMS)-Part II: Treatment and Prevention. Sportverletzung* Sportschaden. 2019;33(01):21-9.

7. Koontz J. The Effects of Common Treatment Modalities on Delayed-Onset Muscle Soreness (DOMS). 2019.

8. Rawson ES, Dolan E, Bryan Saunders ME. Creatine supplementation in sport, exercise and health. Dietary Supplementation in Sport and Exercise: Evidence, Safety and Ergogenic Benefits. 2019:63.

9. Chilibeck PD, Kaviani M, Candow DG, Zello GA. Effect of creatine supplementation during resistance training on lean tissue mass and muscular strength in older adults: a meta-analysis. Open access journal of sports medicine. 2017;8:213.

10. Busanello EN, Marques AC, Lander N, de Oliveira DN, Catharino RR, Oliveira HC, et al. Pravastatin chronic treatment sensitizes hypercholesterolemic mice muscle to mitochondrial permeability transition: Protection by creatine or coenzyme Q10. Frontiers in pharmacology. 2017;8:185.

11. Gonzalez AM. Emerging ergogenic aids for endurance activity and weight loss. Dietary Supplementation in Sport and Exercise: Evidence, Safety and Ergogenic Benefits. 2019:216.

12. Stecker RA, Harty PS, Jagim AR, Candow DG, Kerksick CM. Timing of ergogenic aids and micronutrients on muscle and exercise performance. Journal of the International Society of Sports Nutrition. 2019;16(1):1-8.

13. Brosnan ME, Brosnan JT. The role of dietary creatine. Amino Acids. 2016;48(8):1785-91.

14. Turner CE, Byblow WD, Gant N. Creatine supplementation enhances corticomotor excitability and cognitive performance during oxygen deprivation. Journal of Neuroscience. 2015;35(4):1773-80.

15. Del Favero S, Roschel H, Artioli G, Ugrinowitsch C, Tricoli V, Costa A, et al. Creatine but not betaine supplementation increases muscle phosphorylcreatine content and strength performance. Amino Acids. 2012;42(6):2299-305. 
16. Peeters BM, LANTZ CD, Mayhew JL. Effect of oral creatine monohydrate and creatine phosphate supplementation on maximal strength indices, body composition, and blood pressure. The Journal of Strength \& Conditioning Research. 1999;13(1):3-9.

17. Stout J, Eckerson J, Noonan D, Moore G, Cullen D. Effects of 8 weeks of creatine supplementation on exercise performance and fat-free weight in football players during training. Nutrition Research. $1999 ; 19(2): 217-25$.

18. Ellington WR. Evolution and physiological roles of phosphagen systems. Annual review of physiology. 2001;63(1):289-325.

19. Scheffler TL, Kasten SC, England EM, Scheffler JM, Gerrard DE. Contribution of the phosphagen system to postmortem muscle metabolism in AMP-activated protein kinase $\gamma 3$ R200Q pig Longissimus muscle. Meat science. 2014;96(2):876-83.

20. Bessman SP, Carpenter CL. The creatine-creatine phosphate energy shuttle. Annual review of biochemistry. 1985;54(1):831-62.

21. Felber S, Skladal D, Wyss M, Kremser C, Koller A, Sperl W. Oral creatine supplementation in Duchenne muscular dystrophy: a clinical and $31 \mathrm{P}$ magnetic resonance spectroscopy study. Neurological research. 2000;22(2):145-50.

22. Willer B, Stucki G, Hoppeler H, Brühlmann P, Krähenbühl S. Effects of creatine supplementation on muscle weakness in patients with rheumatoid arthritis. Rheumatology. 2000;39(3):293-8.

23. Vorgerd M, Grehl T, Jäger M, Müller K, Freitag G, Patzold T, et al. Creatine therapy in myophosphorylase deficiency (McArdle disease): a placebo-controlled crossover trial. Archives of Neurology. 2000;57(7):95663.

24. Leuzzi V, Bianchi M, Tosetti M, Carducci C, Cerquiglini A, Cioni G, et al. Brain creatine depletion: guanidinoacetate methyltransferase deficiency (improving with creatine supplementation). Neurology. 2000;55(9):1407-10.

25. Liberati A, Altman DG, Tetzlaff J, Mulrow C, Gøtzsche PC, Ioannidis JP, et al. The PRISMA statement for reporting systematic reviews and meta-analyses of studies that evaluate health care interventions: explanation and elaboration. Annals of internal medicine. 2009;151(4):W-65-W-94.

26. Fedorov S. GetData Graph Digitizer version 2.24. Available at www getdata-graph-digitizer com. 2002;541:542.

27. Higgins JP, Altman DG, Gøtzsche PC, Jüni P, Moher D, Oxman AD, et al. The Cochrane Collaboration's tool for assessing risk of bias in randomised trials. Bmj. 2011;343:d5928.

28. Tobias A. Assessing the influence of a single study in the meta-anyalysis estimate. Stata Technical Bulletin. 1999;8(47).

29. Bassit RA, da Justa Pinheiro CH, Vitzel KF, Sproesser AJ, Silveira LR, Curi R. Effect of short-term creatine supplementation on markers of skeletal muscle damage after strenuous contractile activity. European journal of applied physiology. 2010;108(5):945-55.

30. Robinson TM, Sewell DA, Casey A, Steenge G, Greenhaff PL. Dietary creatine supplementation does not affect some haematological indices, or indices of muscle damage and hepatic and renal function. British journal of sports medicine. 2000;34(4):284-8.

31. Machado M, Pereira R, Sampaio-Jorge F, Knifis F, Hackney A. Creatine supplementation: effects on blood creatine kinase activity responses to resistance exercise and creatine kinase activity measurement. Brazilian journal of pharmaceutical sciences. 2009;45(4):751-7. 
32. Santos R, Bassit R, Caperuto E, Rosa LC. The effect of creatine supplementation upon inflammatory and muscle soreness markers after a 30km race. Life sciences. 2004;75(16):1917-24.

33. Kreider RB, Melton C, Rasmussen CJ, Greenwood M, Lancaster S, Cantler EC, et al. Long-term creatine supplementation does not significantly affect clinical markers of health in athletes. Molecular and cellular biochemistry. 2003;244(1-2):95-104.

34. Cancela P, Ohanian C, Cuitino E, Hackney A. Creatine supplementation does not affect clinical health markers in football players. British journal of sports medicine. 2008;42(9):731-5.

35. Rosene J, Matthews T, Ryan C, Belmore K, Bergsten A, Blaisdell J, et al. Short and longer-term effects of creatine supplementation on exercise induced muscle damage. Journal of sports science \& medicine. 2009;8(1):89.

36. McKinnon NB, Graham MT, Tiidus PM. Effect of creatine supplementation on muscle damage and repair following eccentrically-induced damage to the elbow flexor muscles. Journal of sports science \& medicine. 2012;11(4):653.

37. Veggi KF, Machado M, Koch AJ, Santana SC, Oliveira SS, Stec MJ. Oral creatine supplementation augments the repeated bout effect. International journal of sport nutrition and exercise metabolism. 2013;23(4):378-87.

38. Rawson ES, Gunn B, Clarkson PM. The effects of creatine supplementation on exercise-induced muscle damage. The Journal of Strength \& Conditioning Research. 2001;15(2):178-84.

39. Cooke MB, Rybalka E, Williams AD, Cribb PJ, Hayes A. Creatine supplementation enhances muscle force recovery after eccentrically-induced muscle damage in healthy individuals. Journal of the International Society of Sports Nutrition. 2009;6(1):1-11.

40. Rawson ES, Conti MP, Miles M. Creatine supplementation does not reduce muscle damage or enhance recovery from resistance exercise. Journal of Strength and Conditioning Research. 2007;21(4):1208-13.

41. Kreider RB, Ferreira M, Wilson M, Grindstaff P, Plisk S, Reinardy J, et al. Effects of creatine supplementation on body composition, strength, and sprint performance. Medicine and science in sports and exercise. 1998;30:73-82.

42. Wang C-C, Fang C-C, Lee Y-H, Yang M-T, Chan K-H. Effects of 4-week creatine supplementation combined with complex training on muscle damage and sport performance. Nutrients. 2018;10(11):1640.

43. Cervellin G, Comelli I, Lippi G. Rhabdomyolysis: historical background, clinical, diagnostic and therapeutic features. Clinical chemistry and laboratory medicine. 2010;48(6):749-56.

44. Warren GL, Ingalls CP, Lowe DA, Armstrong R. Excitation-contraction uncoupling: major role in contraction-induced muscle injury. Exercise and sport sciences reviews. 2001;29(2):82-7.

45. Ghaderi M, Rahimi R, Ravasi A, JAAFARI M. The effect of rest interval between sets on markers of muscle damage in professional bodybuilders. Glob J Sci Eng Technol. 2012;3(1):9-15.

46. Rodrigues P, Wassmansdorf R, Salgueirosa FM, Hernandez SG, Nascimento VB, Daros LB, et al. Timecourse of changes in indirect markers of muscle damage responses following a 130-km cycling race. Revista Brasileira de Cineantropometria \& Desempenho Humano. 2016;18(3):322-31.

47. Rahimi MH, Shab-Bidar S, Mollahosseini M, Djafarian K. Branched-chain amino acid supplementation and exercise-induced muscle damage in exercise recovery: A meta-analysis of randomized clinical trials. Nutrition. 2017;42:30-6.

48. Ashtary-Larky D, Lamuchi-Deli N, Milajerdi A, Salehi MB, Alipour M, Kooti W, et al. Inflammatory and biochemical biomarkers in response to high intensity resistance training in trained and untrained men. Asian J Sports Med. 2017;8(2):e13739. 
49. Rawson ES, Miles MP, Larson-Meyer DE. Dietary supplements for health, adaptation, and recovery in athletes. International Journal of Sport Nutrition and Exercise Metabolism. 2018;28(2):188-99.

50. Tack C. Dietary supplementation during musculoskeletal injury: Protein and creatine. Strength \& Conditioning Journal. 2016;38(1):22-6.

51. Willoughby DS, Rosene JM. Effects of oral creatine and resistance training on myogenic regulatory factor expression. Medicine and science in sports and exercise. 2003;35(6):923-9.

52. Brault JJ, Terjung RL. Creatine uptake and creatine transporter expression among rat skeletal muscle fiber types. American Journal of Physiology-Cell Physiology. 2003;284(6):C1481-C9.

53. Mihic S. Effects of Short-Term Creatine Supplementation on Whole-Body Protein Metabolism 2018.

54. Dolan E, Gualano B, Rawson ES. Beyond muscle: the effects of creatine supplementation on brain creatine, cognitive processing, and traumatic brain injury. European Journal of Sport Science. 2019;19(1):114.

55. Gualano B, Rawson ES, Candow DG, Chilibeck PD. Creatine supplementation in the aging population: effects on skeletal muscle, bone and brain. Amino Acids. 2016;48(8):1793-805.

56. Padilha CS, Cella PS, Salles LR, Deminice R. Oral creatine supplementation attenuates muscle loss caused by limb immobilization: a systematic review. Fisioterapia em Movimento. 2017;30(4):831-8.

57. Backx EM, Hangelbroek R, Snijders T, Verscheijden M-L, Verdijk LB, de Groot LC, et al. Creatine loading does not preserve muscle mass or strength during leg immobilization in healthy, young males: a randomized controlled trial. Sports medicine. 2017;47(8):1661-71.

58. Maughan RJ, Gleeson M. The biochemical basis of sports performance: Oxford University Press; 2010.

59. Kutz MR, Gunter MJ. Creatine monohydrate supplementation on body weight and percent body fat. Journal of strength and conditioning research. 2003;17(4):817-21.

60. Juhn MS. Oral creatine supplementation in male collegiate athletes: a survey of dosing habits and side effects. Journal of the Academy of Nutrition and Dietetics. 1999;99(5):593.

61. Earnest CP, Snell P, Rodriguez R, Almada A, Mitchell T. The effect of creatine monohydrate ingestion on anaerobic power indices, muscular strength and body composition. Acta Physiologica Scandinavica. 1995;153(2):207.

62. Terjung RL, Clarkson P, Eichner E, Greenhaff P, Hespel P, Israel R, et al. American College of Sports Medicine roundtable. The physiological and health effects of oral creatine supplementation. Medicine and science in sports and exercise. 2000;32(3):706-17.

63. e Silva AdS, Pertille A, Barbosa CGR, de Oliveira Silva JA, de Jesus DV, Ribeiro AGSV, et al. Effects of creatine supplementation on renal function: a systematic review and meta-analysis. Journal of Renal Nutrition. 2019;29(6):480-9.

64. Aljobaily NA, Viereckl M, Aljobaily HA, Albertson JA, Han Y, Hydock D. Role of Creatine Supplementation in Alleviating Hepatotoxicity Caused by Doxorubicin. The FASEB Journal. 2020;34(S1):1-.

65. Davani-Davari D, Karimzadeh I, Ezzatzadegan-Jahromi S, Sagheb MM. Potential adverse effects of creatine supplement on the kidney in athletes and bodybuilders. Iranian journal of kidney diseases. 2018;12(5):253.

66. Barcelos R, Stefanello S, Mauriz J, Gonzalez-Gallego J, Soares F. Creatine and the liver: metabolism and possible interactions. Mini reviews in medicinal chemistry. 2016;16(1):12-8.

67. Kim J, Kim J. Nutritional Supplement for Athletic Performance: Based on Australian Institute of Sport Sports Supplement Framework. Exercise Science. 2019;28(3):211-20. 
68. Peeling P, Castell LM, Derave W, de Hon O, Burke LM. Sports foods and dietary supplements for optimal function and performance enhancement in track-and-field athletes. International Journal of Sport Nutrition and Exercise Metabolism. 2019;29(2):198-209.

69. Dalbo VJ, Roberts MD, Stout JR, Kerksick CM. Putting to rest the myth of creatine supplementation leading to muscle cramps and dehydration. British journal of sports medicine. 2008;42(7):567-73.

70. Stupka N, Lowther S, Chorneyko K, Bourgeois J, Hogben C, Tarnopolsky M. Gender differences in muscle inflammation after eccentric exercise. Journal of applied physiology. 2000;89(6):2325-32.

\section{Figure legends}

Figure 1. Preferred Reporting Items for Systematic Reviews and Meta-Analyses (PRISMA) flow diagram of study selection process.

Figure 2. Forest plot of the effect of creatine supplementation on CK subgrouped by follow up times after exercise. $\mathrm{WMD}=$ weighted mean difference; $\mathrm{CI}=$ confidence interval.

Figure 3. Forest plot of the effect of creatine supplementation on LDH subgrouped by follow up times after exercise. $\mathrm{WMD}=$ weighted mean difference; $\mathrm{CI}=$ confidence interval.

Figure 4. Funnel plot for evaluating publication bias in CK (A) and LDH (B)

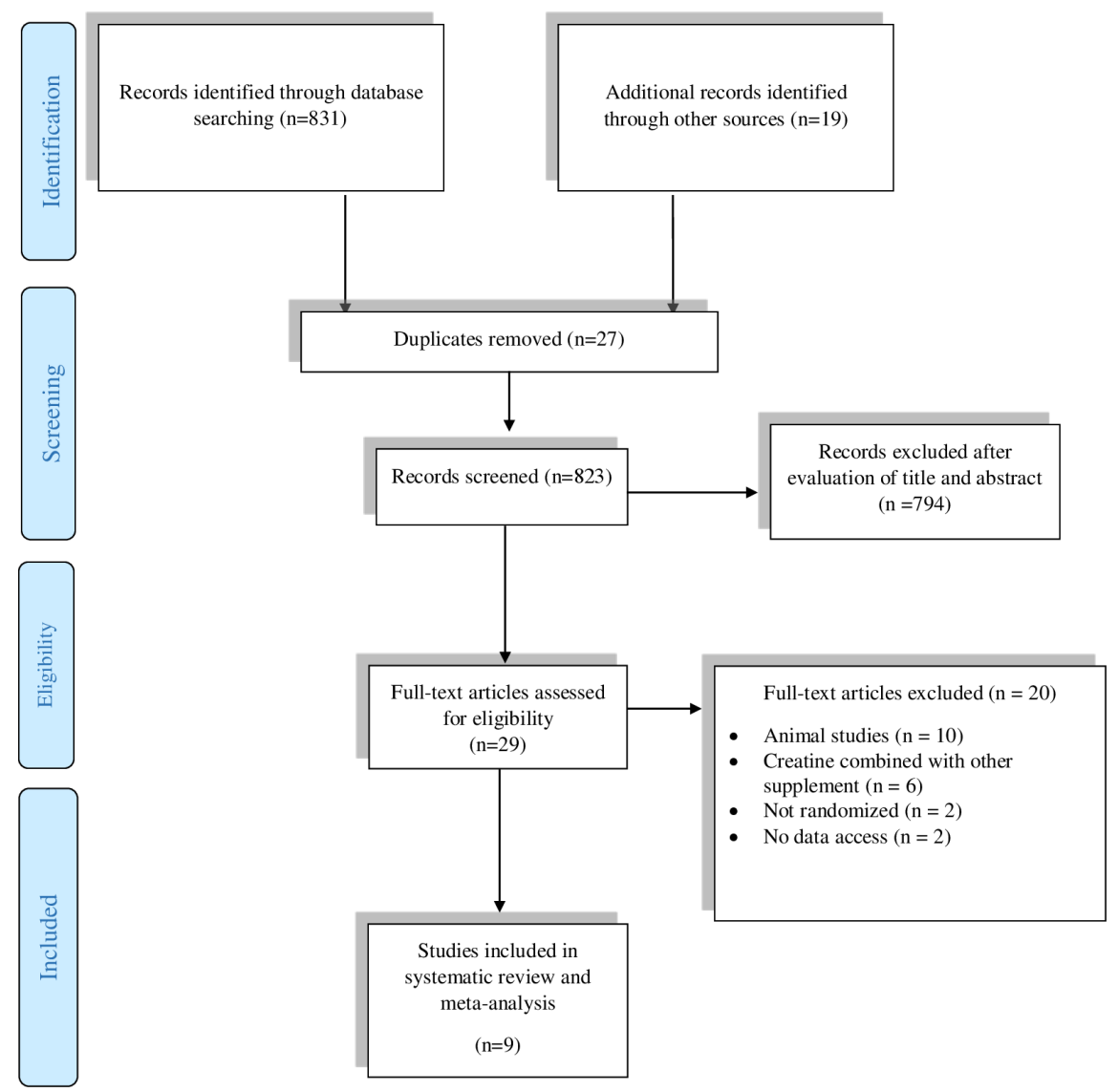




\begin{tabular}{|c|c|c|}
\hline Study First Author & WMD $(95 \%$ CI $)$ & $\%$ Weight \\
\hline $\begin{array}{l}<24 \mathrm{~h} \\
\text { Robinson (2000) } \\
\text { Machado (Men) (2009) } \\
\text { Machado (Women) (2009) } \\
\text { Wang (2018) } \\
\text { Wang (2018) } \\
\text { Subtotal (I-squared }=61.8 \%, \mathrm{p}=0.033 \text { ) }\end{array}$ & $\begin{array}{l}-56.00(-176.19,64.19) \\
64.42(10.31,118.53) \\
-30.56(-55.69,-5.42) \\
-27.97(-78.33,22.40) \\
-11.48(-47.84,24.88) \\
-9.22(-42.71,24.28)\end{array}$ & $\begin{array}{l}2.07 \\
6.20 \\
10.29 \\
6.65 \\
8.59 \\
33.80\end{array}$ \\
\hline $\begin{array}{l}24 \mathrm{~h} \\
\text { Rawson (2001) } \\
\text { Cooke (2009) } \\
\text { Wang }(2018) \\
\text { Wang }(2018) \\
\text { Subtotal (I-squared }=92.6 \%, \mathrm{p}=0.000)\end{array}$ & $\begin{array}{l}477.46(270.62,684.30) \\
-2127.66(-3179.09,-1076.23) \\
-61.73(-123.24,-0.22) \\
-67.40(-119.24,-15.56) \\
9.64(-189.43,208.70)\end{array}$ & $\begin{array}{l}0.79 \\
0.03 \\
5.40 \\
6.47 \\
12.69\end{array}$ \\
\hline $\begin{array}{l}48 \mathrm{~h} \\
\text { Rawson }(2001) \\
\text { Rawson }(2007) \\
\text { Cooke }(2009) \\
\text { Bassit }(2010) \\
\text { Veggi }(2013) \\
\text { Wang }(2018) \\
\text { Wang }(2018) \\
\text { Subtotal (I-squared }=94.5 \%, \mathrm{p}=0.000)\end{array}$ & $\begin{array}{l}-880.48(-1735.12,-25.84) \\
-52.22(-53.38,-51.06) \\
-7446.80(-8899.96,-5993.65) \\
-1015.39(-2015.12,-15.66) \\
-136.36(-678.78,406.06) \\
-11.10(-65.94,43.75) \\
-62.67(-104.64,-20.70) \\
-142.10(-274.79,-9.41)\end{array}$ & $\begin{array}{l}0.05 \\
12.56 \\
0.02 \\
0.04 \\
0.12 \\
6.12 \\
7.77 \\
26.67\end{array}$ \\
\hline $\begin{array}{l}72 \mathrm{~h} \\
\text { Rawson }(2001) \\
\text { Rawson }(2007) \\
\text { Cooke }(2009) \\
\text { Bassit }(2010) \\
\text { Subtotal }(\mathrm{I} \text {-squared }=98.2 \%, \mathrm{p}=0.000)\end{array}$ & $\begin{array}{l}-1179.39(-2346.48,-12.30) \\
0.00(-0.84,0.84) \\
-17765.94(-20672.25,-14859.63) \\
-584.61(-842.24,-326.98) \\
-2611.71(-3889.92,-1333.49)\end{array}$ & $\begin{array}{l}0.03 \\
12.57 \\
0.00 \\
0.52 \\
13.12\end{array}$ \\
\hline $\begin{array}{l}96 \mathrm{~h} \\
\text { Rawson }(2001) \\
\text { Rawson }(2007) \\
\text { Cooke }(2009) \\
\text { Veggi }(2013) \\
\text { Subtotal (I-squared }=97.6 \%, \mathrm{p}=0.000 \text { ) }\end{array}$ & $\begin{array}{l}-761.19(-1881.21,358.83) \\
0.00(-0.84,0.84) \\
-19148.91(-22603.02,-15694.80) \\
-170.46(-341.38,0.46) \\
-1123.15(-1922.77,-323.52)\end{array}$ & $\begin{array}{l}0.03 \\
12.57 \\
0.00 \\
1.12 \\
13.72\end{array}$ \\
\hline $\begin{array}{l}\text { Overall (I-squared }=99.7 \%, p=0.000) \\
\text { NOTE: Weights are from random effects analysis }\end{array}$ & $-28.91(-47.84,-9.98)$ & 100.00 \\
\hline-22603 & & \\
\hline
\end{tabular}




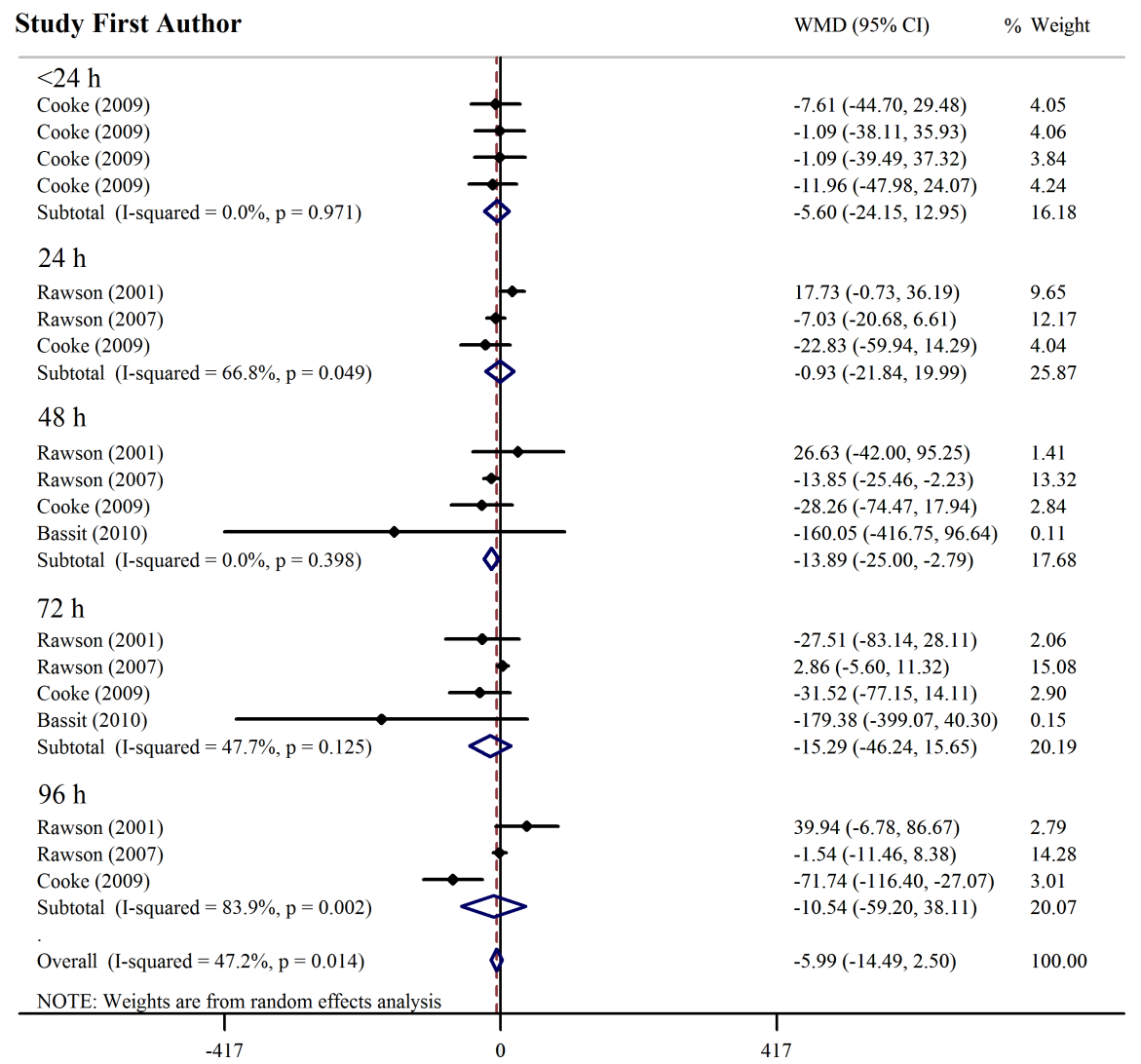
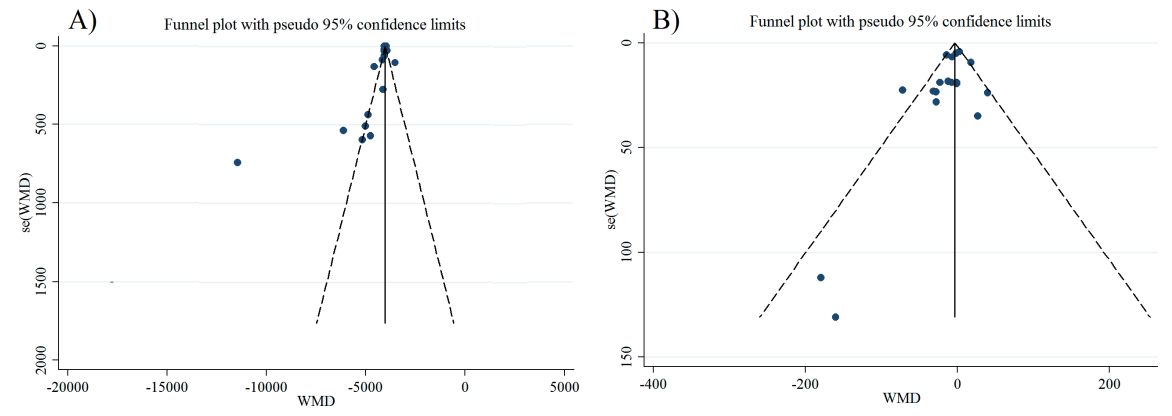

\section{Hosted file}

Table 1. Summary of relevant sources of data.docx available at https://authorea.com/users/ 359187/articles/481372-creatine-supplementation-effect-on-recovery-following-exerciseinduced-muscle-damage-a-systematic-review-and-meta-analysis-of-randomized-controlledtrials

\section{Hosted file}

Table 2. Cochrane Risk of Bias Assessment11.docx available at https://authorea.com/users/ 359187/articles/481372-creatine-supplementation-effect-on-recovery-following-exerciseinduced-muscle-damage-a-systematic-review-and-meta-analysis-of-randomized-controlledtrials 


\section{Hosted file}

Table 3. subgroup CK \& LDH1.docx available at https://authorea.com/users/359187/articles/ 481372-creatine-supplementation-effect-on-recovery-following-exercise-induced-muscledamage-a-systematic-review-and-meta-analysis-of-randomized-controlled-trials 